\title{
Value and impact of necropsy in paediatric cardiology
}

\author{
Michael A Gatzoulis, Mary N Sheppard, Siew Yen Ho
}

\begin{abstract}
Objective-To assess the current value of necropsy in paediatric cardiology and cardiothoracic surgery and determine its potential impact on clinical practice.

Design and setting-Descriptive study of all paediatric cardiac deaths occurring over four years in a tertiary referral centre. Data were obtained from reviewing the hospital files, available necropsy records and specimens, and audit reports.

Patients-Paediatric patients with congenital or acquired heart disease, who died of a cardiac cause between January 1992 and July 1995 . Inclusion criteria were that the diagnosis of heart disease was made before death, and that patients were managed thereafter medically and/or surgically at the referral centre. The value of necropsy was assessed according to its contribution in establishing the cause of death (confirmed, clarified, precise cause of death uncertain) and the anatomy(simple confirmation or additional information provided). For cases not submitted to necropsy the clinical information relating to the cause of death was assessed and the case assigned as cause of death firm, uncertain, or unknown.

Results-One hundred and six deaths were identified (61 males, age at death: one day to 20 years). Seventy occurred early (a month or less) after surgery and were graded as postoperative deaths. The
\end{abstract}

Table 1 Clinical characteristics of cases with and without a necropsy

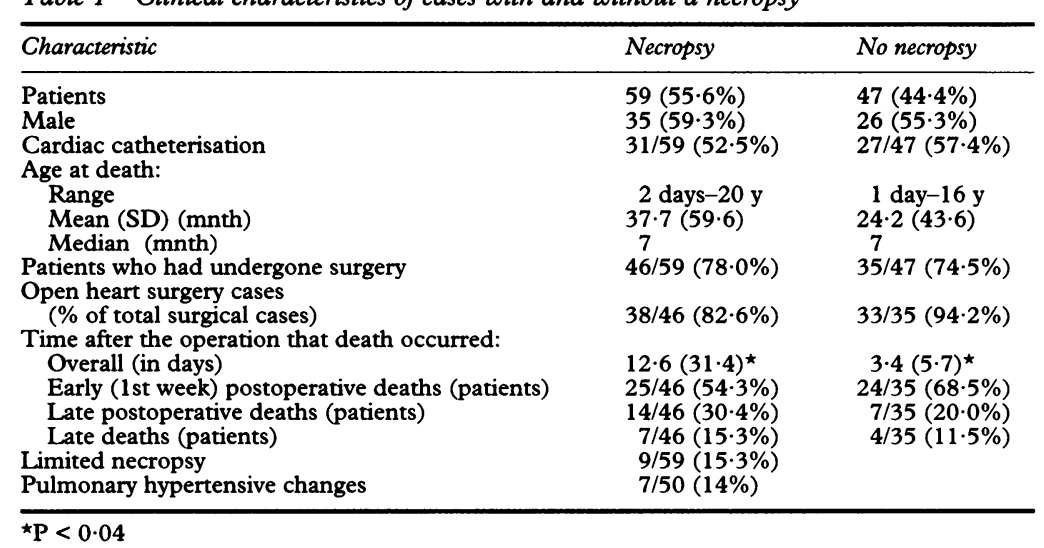

rest were considered to be either medical or late surgical deaths. Necropsy was performed in $59(55 \cdot 6 \%)$. The precise cause of death was confirmed in $33(55.9 \%)$, clarified in $22(37 \cdot 3 \%)$, and remained uncertain in four $(\mathbf{7 \cdot 8 \%})$. Additional information regarding the anatomy was found in eight $(13.6 \%)$ cases. In five cases $(8 \cdot 5 \%)$ the necropsy detected findings which, if known before death, would probably have improved outcome. For the patients dying without a necropsy, the cause of death remained uncertain in 10 $(21 \cdot 3 \%)$ and unknown in seven $(14 \cdot 9 \%)$. In $36 \%$ of cases, therefore, a firm cause of death that might have been provided by a necropsy was missing.

Conclusion-In paediatric cardiology necropsy continues to provide clinically relevant information at a high level. It remains vital for ensuring quality of medical care, in instigating improvements in future management, and increasing understanding of congenital heart disease. The procedure should therefore be sought actively in all cases.

(Heart 1996;75:626-631)

Keywords: necropsy; heart disease; congenital; heart surgery

The primary role of necropsy in paediatric cardiology is to investigate the cause of death and establish the precise diagnosis. ${ }^{12}$ As an additional source of information, it may challenge or verify the impact of new diagnostic techniques, monitor effects of treatment, facilitate parental counselling, and form a basis for research and education. ${ }^{3-6}$ Relevant data concerning paediatric necropsies are surprisingly lacking. Several issues may have had an adverse effect on recent rates of necropsythese include the belief that the diagnostic yield has been diminished by improved technology, a fear of litigation resulting from discrepancies between clinical and necropsy diagnoses, financial restraints, and problems with obtaining consent.

The purpose of this study was to assess the value of paediatric necropsy in the current era of advanced diagnostic technology and sophis- 
ticated surgery for complex congenital and acquired heart disease. We also sought to determine its potential impact on clinical practice.

\section{Patients and methods}

We studied all patients treated in the department of paediatrics at the Royal Brompton Hospital with congenital or acquired heart disease who died from a cardiac cause between

Table 2 Cases in which necropsy disclosed additional anatomical findings

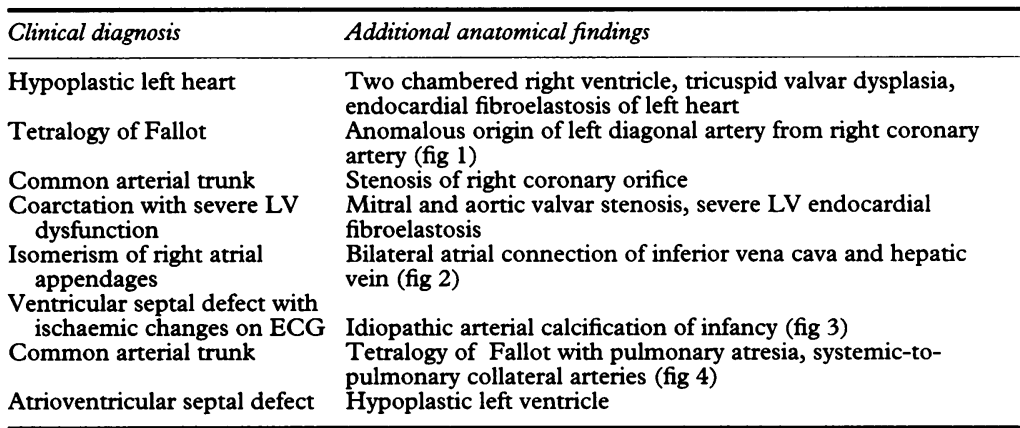

LV, left ventricular; ECG, electrocardiogram.

Table 3 Clinical diagnoses and scores following the evaluation process in cases with and without necropsy

\begin{tabular}{|c|c|c|}
\hline & $\begin{array}{l}\text { Necropsy } \\
\text { (59) }\end{array}$ & $\begin{array}{l}\text { No necropsy } \\
\text { (47) }\end{array}$ \\
\hline Atrial or ventricular septal defect & $2(\mathrm{BE})$ & 3 \\
\hline Atrioventricular septal defect & 7 (BBBBE) & $5(\mathrm{bc})$ \\
\hline Tetralogy of Fallot (Pulmonary stenosis and atresia) & 8 (BBBCE) & 2 (c) \\
\hline Double inlet ventricle & & 3 (b) \\
\hline Double outlet ventricle & $6(\mathrm{BBC})$ & 3 (bc) \\
\hline Hypoplastic left ventricle & $6(\mathrm{BE})$ & 6 (b) \\
\hline Hypoplastic right ventricle & $4(\mathrm{BBC})$ & $3(\mathrm{bb})$ \\
\hline Complete transposition & $2(\mathrm{BC})$ & 3 (b) \\
\hline Congenitally corrected transposition & 3 (B) & $2(\mathrm{cc})$ \\
\hline Isomeric atrial appendages & 5 (BE) & 3 (bc) \\
\hline Common arterial trunk & 2 (EE) & 2 \\
\hline \\
\hline $\begin{array}{l}\text { (Aortic coarctation/interruption, Aortopulmonary window, } \\
\text { coronary arterial abnormalities) }\end{array}$ & $2(\mathrm{BE})$ & 3 (bc) \\
\hline \multirow{2}{*}{\multicolumn{3}{|c|}{$\begin{array}{l}\text { Miscellaneous } \\
\text { (obstructed systemic ventricular inflow, cardiomyopathies, }\end{array}$}} \\
\hline & & 9 (b) \\
\hline
\end{tabular}

$B$, cause of death was clarified only after necropsy; $C$, precise cause of death remained uncertain despite necropsy; $E$, additional anatomical information found at necropsy; $b$, cause of death uncertain (no necropsy); c, cause of death unknown (no necropsy).

Each letter represents an individual case. $A \mathrm{~s}, D \mathrm{~s}$ and $a$ are not included (see text).
January 1992 and July 1995. The necessary detailed data had been available during this time from our regular clinico-pathological audit meetings. This period is also representative of the latest era, incorporating the most recent advances such as transoesophageal echocardiography, which has been shown to improve our diagnostic capability, particularly in postoperative patients. ${ }^{7}$ Inclusion criteria were that the diagnosis of heart disease was made before death, and that patients were managed medically and/or surgically thereafter at our hospital. Data were obtained from hospital files, necropsy records, and audit reports and reviewed independently by two investigators. Most of the heart specimens were available for re-examination should any points have remained unclear after the initial report. The value of necropsy was assessed according to its contribution to establishing the cause of death in conjunction with the clinical data. Cases were grouped according to diagnosis: A confirmed, B clarified/found, and C precise cause of death uncertain. Clarification of the cardiac anatomy was scored as: D simple confirmation of the anatomy or $\mathrm{E}$ additional information found. The two investigators, blinded from each other, also examined each case to see whether clinical outcome could have been improved had the data provided by the necropsy been available before death. Cases were regarded as positive only if both investigators concurred. For cases without a necropsy the clinical information and the audit reviews relating to the cause of death were assessed in the same way and the cases assigned to three groups: cause of death a firm, b uncertain, and c unknown. When there was disagreement between the two reviewers, the lower score was taken so as to ensure that the value of necropsy was not overstated. Student's $t$ test was used to identify differences in clinical characteristics between patients with and without necropsies.

\section{Results}

We identified 106 deaths. Seventy (66\%) of them occurred in the hospital within one month of a surgical procedure. The rest occurred in
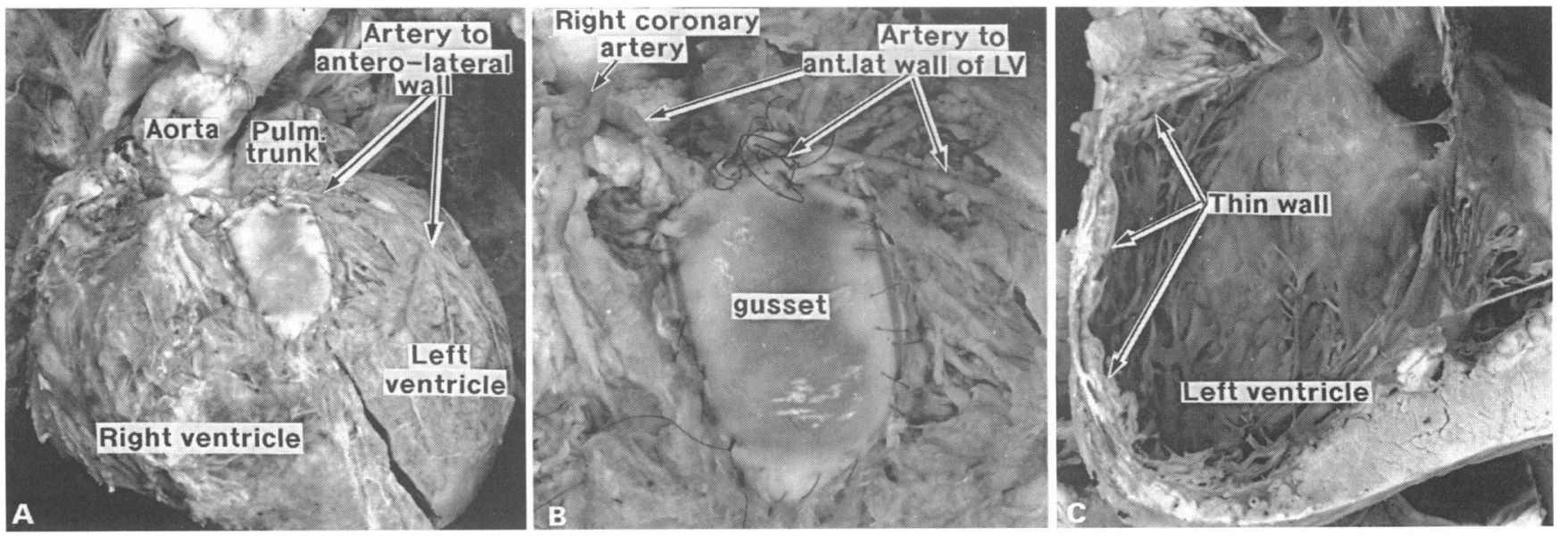

Figure 1 This three year old child underwent repair for tetralogy of Fallot. She had a prolonged and complicated postoperative course with poor ventricular function. (A) Overall view shows a gusset of Impra enlarging the right ventricular outflow tract. (B) $A$ close-up view shows a coronary artery passing along the upper margin of the gusset. This artery arises from the right coronary artery to pass diagonally across the antero-lateral wall of the left ventricle. (C) The antero-lateral wall of the left ventricle is fibrotic and thin because of ischaemic changes after injury to the diagonal artery. 
Figure 2 This two year old child with isomeric right atrial appendages had total cavopulmonary anastomosis with a fenestrated intra-atrial baffle. Her postoperative coursewas complicated by progressive cyanosis and arrhythmia. (A) There is isomeric arrangement of the right atrial appendages. (B) The intra-atrial baffle is positioned in the right-sided atrium. (C) This longitudinal section shows both atrial chambers of right morphology opening through a common valve to a morphologically right ventricle (double inlet ventricle). The intra-atrial baffle has been removed. The suture-line (dots) encircles the orifice of the inferior vena cava but the orifice of the left hepatic vein remains within the pulmonary venous atrium, leading to a right-to-left shunt.
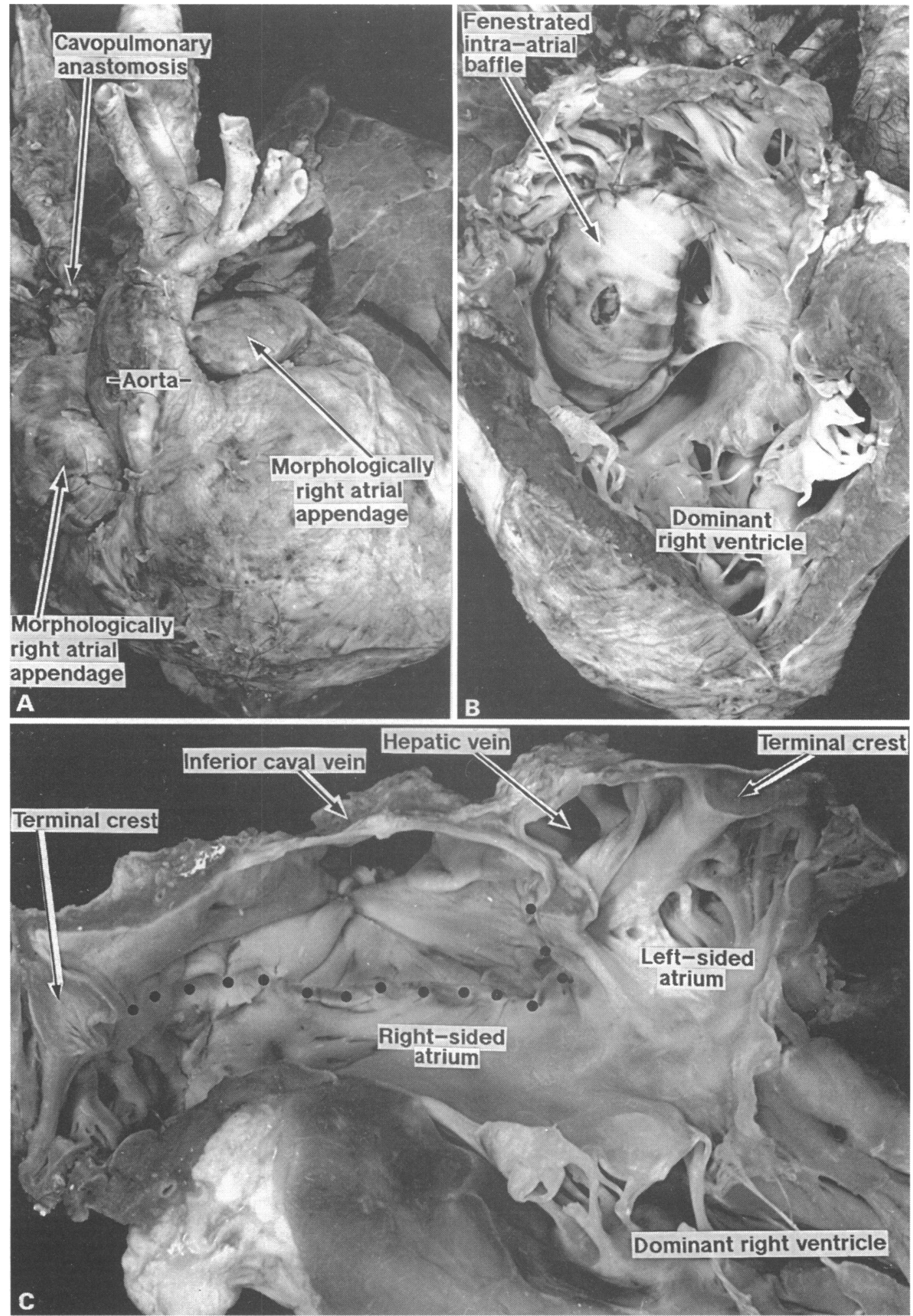

patients who had previously undergone surgery or were purely medical cases. For all cases an early diagnosis had been established by echocardiography after physical examination. Some patients had had cardiac catheterisation and/or other diagnostic examinations. Although permission for necropsy is routinely sought in our hospital, necropsy was performed in only $59 / 106(55.6 \%)$ cases. In 40 cases, and within the limitations of a retrospective study, we were able to identify the reason why a necropsy was not performed. In $34(85 \%)$ the family declined consent, in five $(12.5 \%)$ necropsy was not requested, and, in one $(2.5 \%)$, the family could not be contacted to obtain immediate consent.

Table 1 shows the clinical characteristics of the patients with and without necropsy.

The only difference between these two groups was that the postoperative stay was longer in the necropsy group.

The two investigators gave a different score in three cases: the lower score was used.

The precise cause of death was confirmed in $33(55.9 \%)$, clarified in $22(37 \cdot 3 \%)$, and remained uncertain in four $(6 \cdot 8 \%)$. Additional information regarding the anatomy was found in eight cases (13.6\%, table 2 and figs $1-4)$. Table 3 shows the clinical diagnoses and scores 
Figure 3 Nine week old infant with a ventricular septal defect and impaired ventricular function: $(A)$ Overall view of the heart specimen. There are white patches along the length of the major right and left coronary arteries. (B)

Right ventricular view

shows a moderate-sized perimembranous

ventricular septal defect. (C) The left ventricle shows fibrous streaks

(arrows) and occlusion of the circumflex artery (open arrow). (D) Histological sections of the left coronary artery. The upper panel shows reduction of the lumen due to intimal hyperplasia with calcification at the site of the internal elastic membrane. The lower panel shows total occlusion of the lumen with small foci of chronic inflammation.

Calcification affected some of the pulmonary arteries but was not seen in the walls of the great arteries or neck and arm arteries. The diagnosis is idiopathic diagnosis is idiopathic
calcification of infancy.
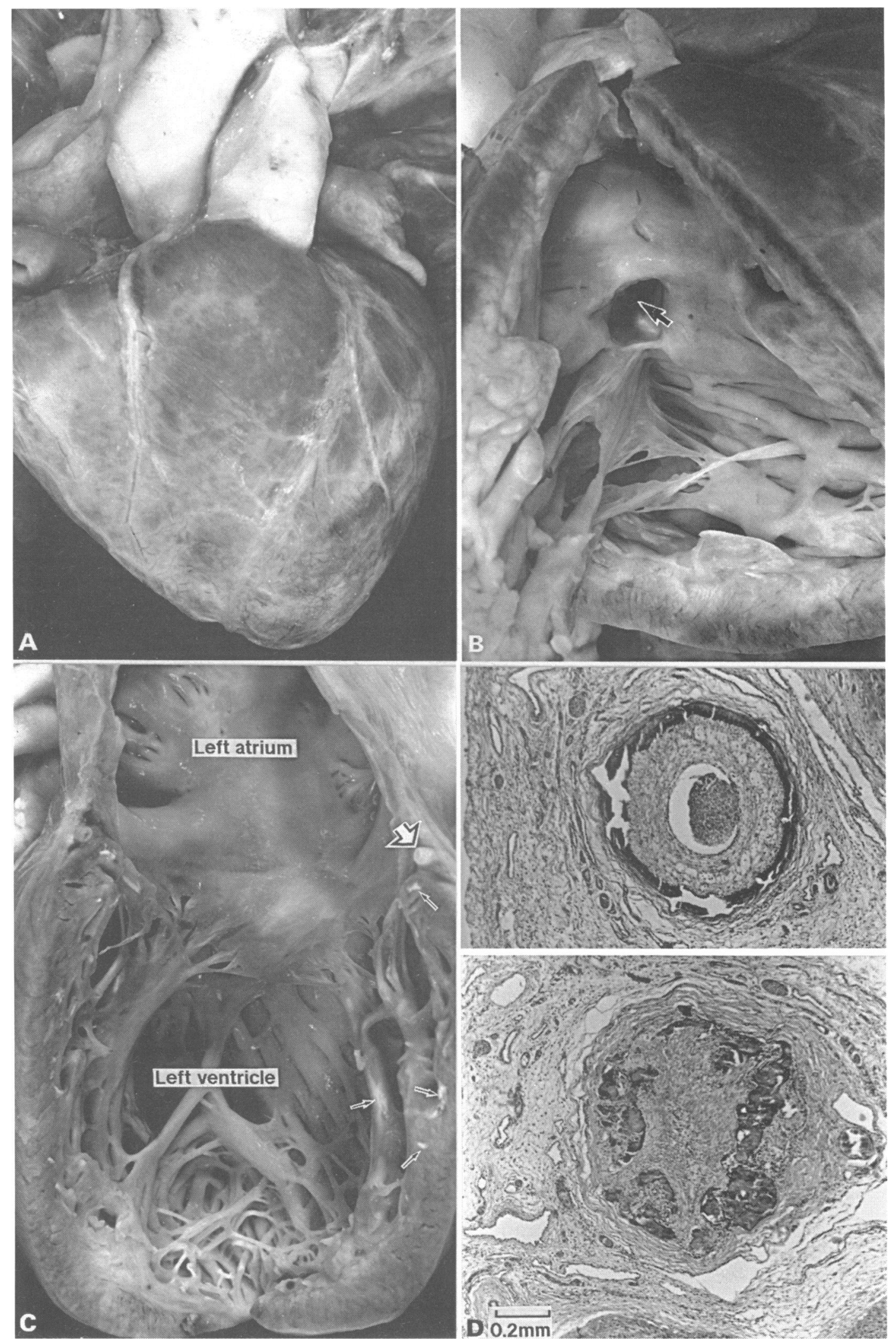

after the evaluative process. In five $(8.5 \%)$ cases (table 4 ) both investigators agreed that the necropsy detected findings that had they been known before surgery or death would probably have improved outcome. For the patients dying without a necropsy, the cause of death remained uncertain in $10(21.3 \%)$ and unknown in seven $(14.9 \%)$. In $36.2 \%$ of cases, therefore, a firm cause of death, that might have been disclosed by a necropsy, was not known.

\section{Discussion}

In paediatric cardiology necropsy has always been recognised as the final audit on which to base the precise cause of death and to establish the definitive anatomical diagnosis. Although this role today has largely remained unchanged, there has been some shift in emphasis. $^{8}$

Better understanding of congenital heart disease and recent advances in diagnostic techniques have led to a complete diagnosis 
Figure 4 This preterm infant presented soon after birth in heart failure.

Echocardiography showed a heart with a solitary arterial trunk, a dysplastic and regurgitant arterial valve, a large ventricular septal defect, and no intrapericardial pulmonary arteries. (A) Ascending aortogram showing multiple systemicvessels to both right and left lungs. No

intrapericardial pulmonary arteries are seen. (B) There is atresia of the ventricular origin. Although the lumen of the trunk is patent, it is too echocardiography or angiography. The complete diagnosis, therefore, is tetralogy of Fallot with pulmonary atresia. This could be established only by direct inspection of the area either during surgery (unsuitable) or necropsy. (C) The pulmonary trunk connects only to the right pulmonary artery. The lefi pulmonary artery arises from the aorta via the arterial duct.

(D) Systemic-to-pulmonary collateral arteries from the descending aorta supply part of the right lung. pulmonary collateral pulmonary trunk at its tiny to be detected either by
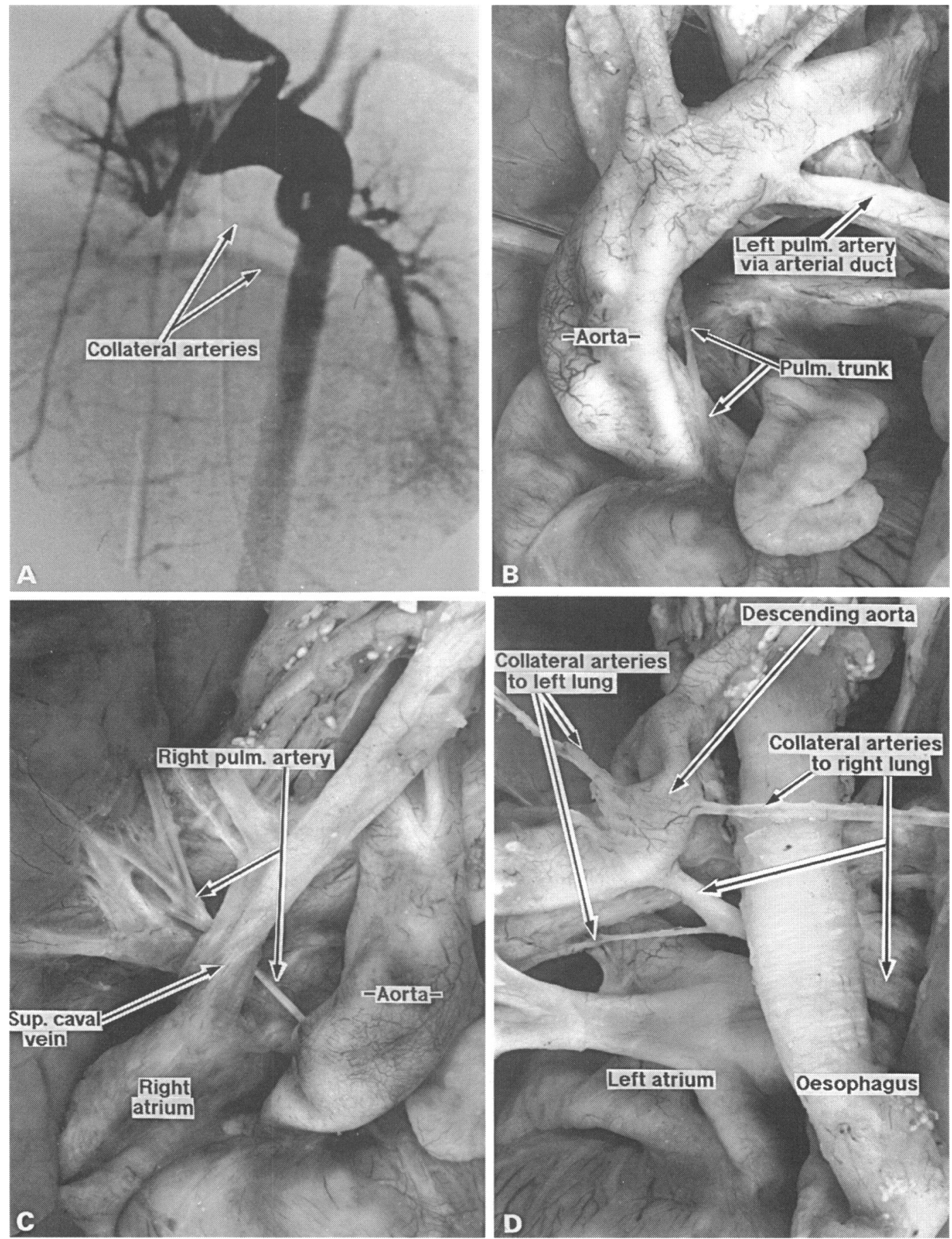

being reached in most cases before death, a trend confirmed by our study. There is still a great need to establish the precise cause of death and monitor the effects of treatment. Now that complex cardiac surgery and newly developed transcatheter techniques are increasingly used for infants or children with

Table 4 Cases with necropsy findings that if known before surgery or death could have altered clinical outcome

\begin{tabular}{llll}
\hline Case & Operation & Postoperative problem & Necropsy finding \\
\hline IRAA & TCPC & Cyanosis & R to L shunt/hepatic collateral \\
TOF & Homograft repair & Poor function & LAD from RCA \\
TOF & T/P repair & Poor function & 2nd LAD from RCA \\
TOF & Repair & Persistent effusions & Purulent pericarditis \\
VSD/Down's & Surgical closure & ARDS picture & Pseudomonas (multiple abscesses) \\
\hline
\end{tabular}

IRAA, isomerism of right atrial appendages; TCPC, total cavopulmonary anastomosis $\mathrm{R}$, right; $\mathrm{L}$, left; TOF, tetralogy of Fallot; LAD, left anterior descending coronary artery, RCA, right coronary artery; T/P, transannular patch; VSD, ventricular septal defect; ARDS adult respiratory distress syndrome. complex congenital heart disease this need is greater than before.

Necropsy rates in our centre were relatively high. ${ }^{910}$ This is welcome, but not surprising for a paediatric tertiary referral centre where complex cardiac surgery is performed routinely. The availability of advanced diagnostic techniques, it has been argued, might reduce the value of necropsy. ${ }^{11}$ Transoesophageal echocardiography, for example, has been shown to improve diagnostic yields, particularly in postoperative patients. ${ }^{7}$ In many cases in this study the precise cause of death remained uncertain or even unknown before necropsy, despite the wide use of newer diagnostic techniques. Necropsy, when performed, nearly always confirmed or clarified the precise cause of death. Furthermore, necropsy revealed a number of unexpected anatomical findings, some 
of which, if they had been known before death, could have altered the clinical outcome. Not surprisingly, abnormal coronary arteries or collateral vessels were the most common findings, as they can be difficult to visualise. A modified diagnostic approach ${ }^{12}$ and awareness of and prompt response to postoperative complication ${ }^{13}$ have improved both our diagnostic capability and overall outcome. In seven (4\%) of the 50 cases, histological findings in the lungs were consistent with pulmonary hypertensive changes, which clinically were thought to have contributed to death. Because cardiopulmonary interactions are so common in children after operative procedures involving the heart, this is an area that should be investigated routinely, whenever a necropsy is performed.

Our data show that necropsy with detailed examination of the heart and lungs remains the ultimate investigation for establishing the precise cause of death in most cases, either by confirming the presumed clinical cause of death or establishing one. The criteria for assessing the value of necropsy, however, must be broader than merely confirming anatomical diagnoses or-in conjunction with the clinical data-establishing the cause of death. Necropsy findings, even when negative, are important in reassuring parents and clinicians that important disease has not been missed. Furthermore, scrutiny of highly skilled, complex operative interventions on hearts at necropsy is essential for identifying possible shortfalls in the attempts of clinicians to produce a perfect result. Necropsy can guide clinicians in optimising some of the technical aspects of newly introduced forms of treatment.

There can be difficulties in obtaining consent for necropsy. ${ }^{14}$ There is no euphemism to describe "yet another investigation" to the parents of a child who dies in an intensive care unit, often after major heart surgery. We found that patients who subsequently came to necropsy were more likely to be those who had stayed considerably longer in intensive care after operation. This contrasts with a previous report. ${ }^{3}$ One reason for this difference may be that, in these cases, the clinicians involved were more keen to elucidate the cause of death. In our experience, a simple and clear explanation why a necropsy is being sought, given by a senior member of staff who has already established rapport and understanding with the family, is almost always successful.

We conclude that in paediatric cardiology necropsy continues to provide clinically useful information at a high level. In striving to ensure top-quality medical care, for further improvement in future management, and for increasing our understanding of congenital heart disease, necropsy should continue to be sought actively in all cases.

We thank all our paediatric cardiac and cardiothoracic surgica colleagues for allowing us to report these cases and for their continuous support with the process of review. We also than our colleagues in the Department of Histopathology, Roya Brompton Hospital, who performed many of the general necropsies and the many pathologists in peripheral hospitals who made specimens available for our evaluation. We are indebted to all research fellows for their active participation in the mortality/audit meetings. Finally, we are grateful to Prof $R H$ Anderson for his helpful comments during the preparation of this paper. SYH is supported by the British Heart Foundation.

1 Russell GA, Berry PJ. Postmortem audit in a paediatric cardiology unit. $f$ Clin Pathol 1989;42:912-8.

2 Scottollini AG, Weinstein SR. The autopsy in clinical quality control. $\mathcal{F} A M A 1983 ; 250: 1192-4$.

3 Landefeld CS, Chren MM, Myers A, Geller R, Robbins S, Goldman L. Diagnostic yield of the autopsy in a university hospital and a community hospital. $N$ Engl $f$ Med 1988; 318:1249-54.

4 Kay MH, Moodie DS, Sterba R, Murphy DJ, Rosenkranz $\mathrm{E}$, Ratliff N, et al. The value of the autopsy in congenital E, Ratliff N, et al. The value of the autopsy

5 Hegerty AS, Anderson RH, Ho SY. Congenital heart malformations in the first year of life- a necropsy study. $\mathrm{Br}$
fort Heart $\mathcal{F} 1985 ; 54: 583-92$.

6 Cartlidge PHT, Dawson AT, Stewart JH, Vujanic GM Value and quality of perinatal and infant postmortem examinations: cohort analysis of $\mathbf{4 0 0}$ consecutive deaths. BMF 1995;310:155-8

7 Stumper O, Kaulitz R, Elzenga NJ, Bom N, Roelandt JR, Hess J, et al. The value of transesophageal echocardiography in children with congenital heart disease. $\mathcal{F} \mathrm{Am}$ Soc Echocardiogr 1991;4:164-76.

8 Anderson RH, Ho SY. The postoperative pathology of congenital heart disease. Cardiol Young 1993;3:147-57.

9 Stambouly J, Kahn E, Boxer RA. Correlation between clinical diagnoses and autopsy findings in critically ill children. Pediatrics 1993;92:248-51.

10 Geller SA. Autopsy. Sci Am 1983;248:124-36.

11 Goldman L, Sayson R, Robbins S, Cohn L, Bettman M, Weisberg $M$. The value of autopsy in three medical eras. $N$ Engl f Med 1983;308:1000-5.

12 Carvalho JS, Silva CMC, Rigby ML, Shinebourne EA Angiographic diagnosis of anomalous coronary artery in tetralogy of Fallot. Br Heart $\mathcal{f} 1993 ; 70: 75-8$.

13 Gatzoulis MA, Shinebourne EA, Redington AN, Rigby MI, Ho SY, Shore DF. Increasing cyanosis early after cavopulmonary connection caused by abnormal systemic venous channels. Br Heart $\mathcal{F}$ 1995; 73:182-6.

14 VanMarter LJ, Taylor F, Epstein MF. Parental and physician-related determinants of consent for neonatal autopsy. Am F Dis Child 1987;141:149-53. 